\title{
THE PREVALENCE OF INTESTINAL PARASITES IN AMMAN, JORDAN
}

A. M. Chazal and H. K. Adi

Faculty of Pharmacy, Al-Isra Private University, Amman, Jordan

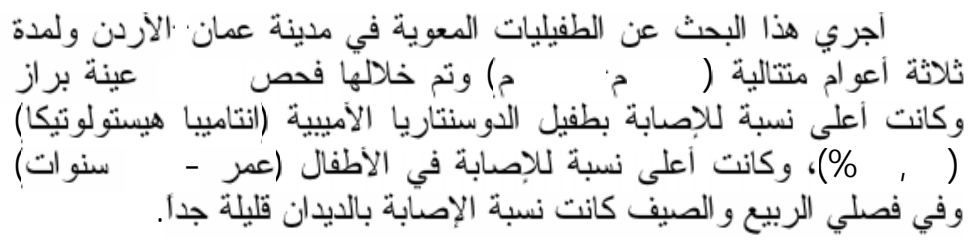

Intestinal parasitic infections were investigated in Amman, Jordan, during the period extending from 2003 until 2005. A total number of 1280 specimens were included in the study. Entamoeba histolytica was found to be the most prevalent parasite with an infection rate of $27.81 \%$.

The highest incidence of this infection was during spring and summer seasons and at an early age (1-10 years). Helminthic infections were uncommon during this survey.

\section{INTRODUCTION}

Although mortality from intestinal parasitic infections is relatively low, complications are not uncommon and many cases may need hospital care.

In many countries, malabsorption, diarrhea, blood loss, impaired work capacity, and reduced growth rate due to intestinal parasitic infections constitute important health and social problems ${ }^{1}$.

The predominant parasites, vary in different geographical areas.
Recent published data on the prevalence of intestinal parasites in Amman, Jordan is scarce.

This study was aimed at determining the intestinal parasites found in this part of the world, their seasonal occurrence, and their prevalence in relation to age and sex of infected individuals.

\section{MATERIAL AND METHODS}

Most of the patients were referred from clinics to a private medical laboratory, in Amman, Jordan for a stool examination. 
It was part of a routine examination of patients complaining of intestinal disorders.

Samples were examined macroscopically for worms, proglottids or blood, mucous and microscopically for parasites. Both direct and concentration methods were used in the study.

Wet mount of each specimen was done by using physiological saline and lugol's iodine solution on a microscopic slide.

The scotch tape technique was used in children whenever suitable to detect eggs or worms of Enterobius vermicularis.

\section{RESULTS AND DISCUSSION}

The study period extended for three years, from 2003 until 2005. 1280 stool samples were examined of which 378 samples had positive findings (29.5\%) (Table 1).

Entamoeba histolytica was the most frequent intestinal parasites with an infection rate of $27.81 \%$ totaling 356 out of 1280 stool samples examined (Table 1 and Figure 1) $E$. histolytica made up $94.17 \%$ of all positive cases, totaling 356 out of the 378 positive cases (Table 1 and Figure 1).

Giardia lamblia comprised the second most frequent intestinal parasites (13 cases) (Table 1 and Figure 1).

Low incidence of Ascaris lumbricoides, vermicularis, Enterobius stercoralis and Trichomonas hominis was noted $(3,2,2 \quad \& \quad 2$ cases consequently) (Table 1 and Figure 1).

Higher incidence of infections with Entamoeba histolytica was noted in spring \& summer (104 and 108 infection consequently) as compared to those in autumn and winter $(77 \&$ 67 infection consequently) as shown in Figure 2.

The sex distribution did not have an influence on the rate of infection (Figure 3).

However, an age related incidence of amoebiasis was established where a relatively higher frequency of infection was noted in children compared to older age groups (Figure 4).

The number of infected children aged between 1 and 10 years was 171 out of a total $356(48 \%)$ and 39 patients among those aged 11 to 20 years $(10.95 \%)$.

Amoebiasis has a world wide distribution and it is more prevalent in tropical and subtropical areas than elsewhere ${ }^{2}$.

This finding applies to this region of Jordan (Amman) where we found amoebiasis as the most prevalent intestinal infection.

These findings are consistent with another study in Amman area ${ }^{3}$, while they disagree with the findings obtained in northern Jordan (Irbid) ${ }^{4 \& 5}$.

In other parts of the world where there is different climates, the prevalence rates of amoebiasis was different.

High incidence of amoebiasis was also found in other middle eastern countries like sudan ${ }^{6}$ and lebanon ${ }^{7}$. 
Table 1: The prevalence of intestinal parasites in patients with different age group.

\begin{tabular}{|l|c|c|c|}
\hline \multirow{2}{*}{} & \multicolumn{3}{|c|}{ Total number examined =1280 } \\
\cline { 2 - 4 } & $\begin{array}{c}\text { Number of positive } \\
\text { cases }\end{array}$ & $\begin{array}{c}\% \\
\text { positives }\end{array}$ & $\begin{array}{c}\text { Age } \\
\text { (years) }\end{array}$ \\
\hline E.histolytica & 356 & 27.81 & $1-70$ \\
\hline G.lamblia & 13 & 1.01 & $1-30$ \\
\hline A.Lumbricoides & 3 & 0.23 & $3-5$ \\
\hline E.Vermicularis & 2 & 0.15 & $1-12$ \\
\hline S.stercoralis & 2 & 0.15 & $33-38$ \\
\hline T.hominis & 2 & 0.15 & $20-25$ \\
\hline Total number of positives & 378 & 29.5 & \\
\hline
\end{tabular}

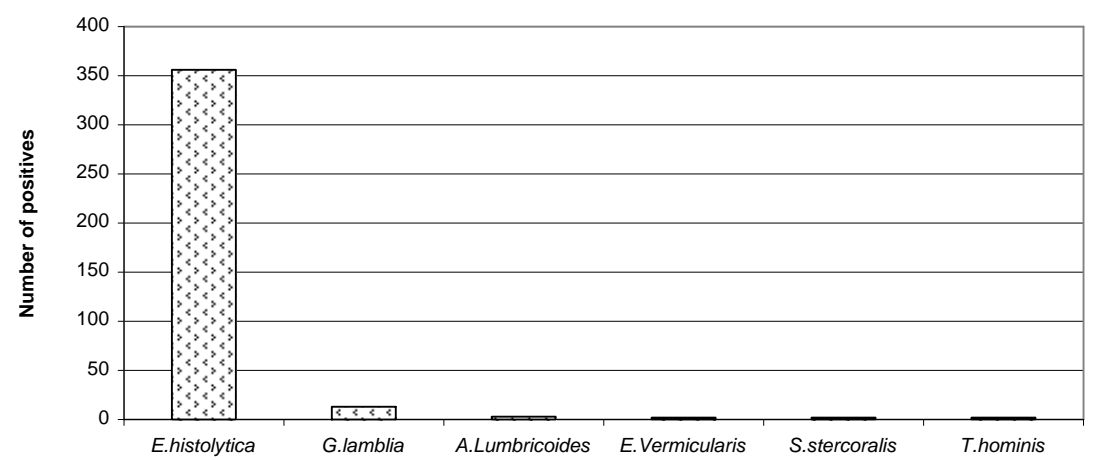

Fig. 1: The numbers of positive findings in stool examination of the detected intestinal parasites.

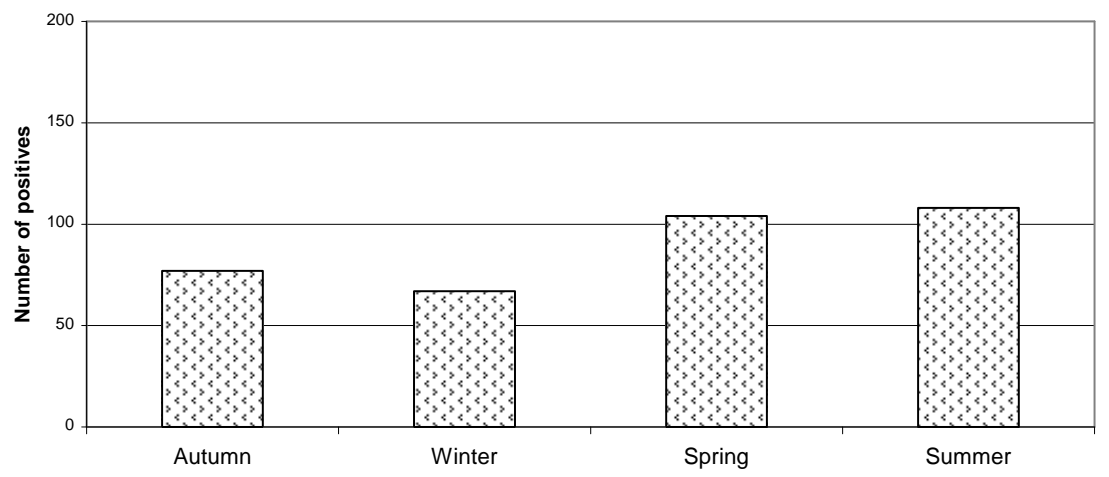

Fig. 2: Seasonal distribution of Entamoeba histolytica infection. 


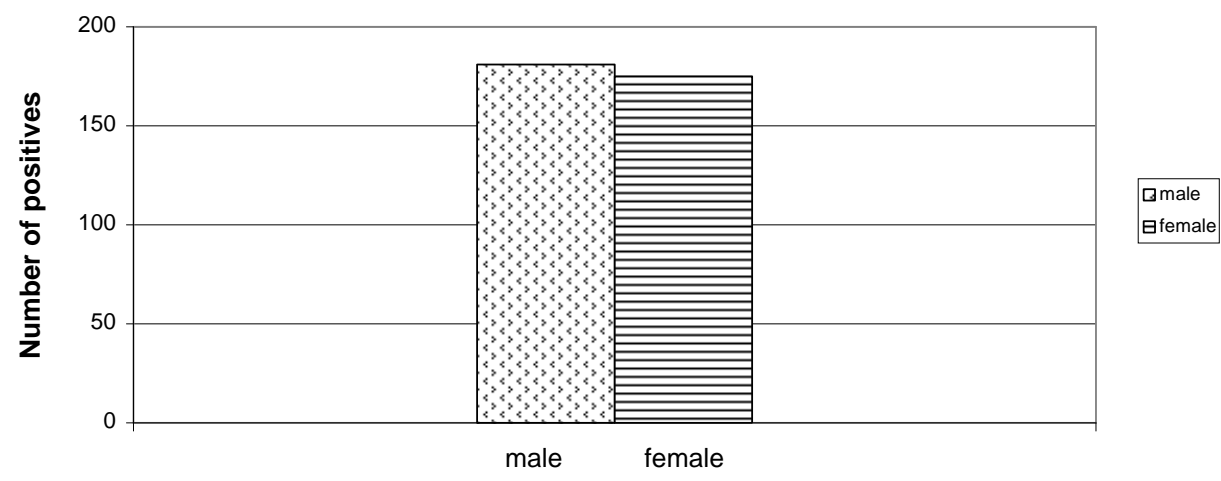

Fig. 3: Sex distribution of E.histolytica infection.

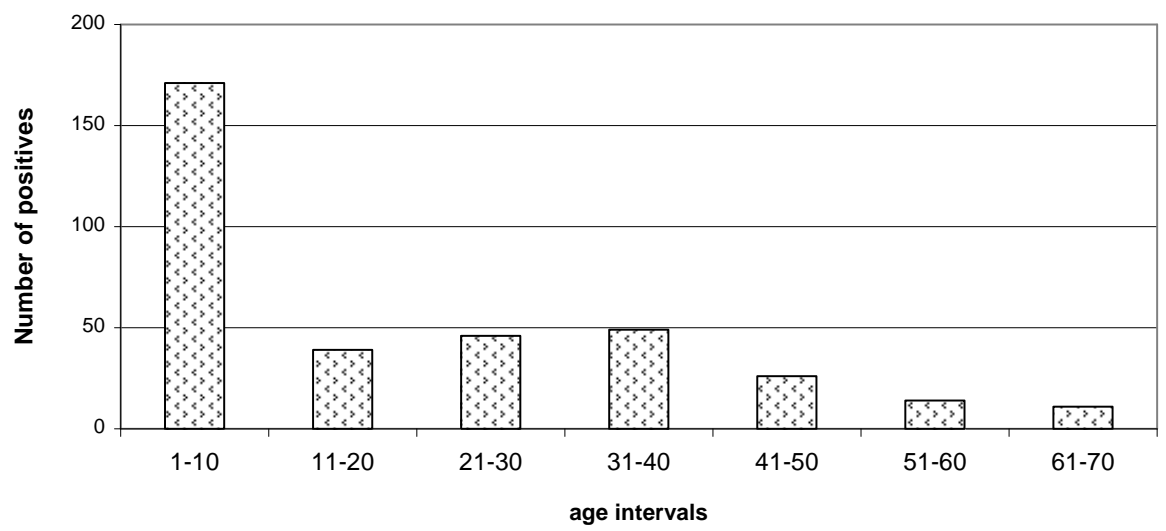

Fig. 4: The incidence of infection with Entamoeba histolytica according to age, in years, as patient been divided to seven intervals.

Lower incidence, however was found in Saudi Arabia ${ }^{8}$ \& Iraq 9 .

Low incidence of infection with Giardia lamblia we found in our results coincide with the results of others in Jordan ${ }^{4 \& 5}$.

Very low incidence of helminthes infection in our results disagree with a previous study in $\mathrm{Amman}^{3}$. This may be explained as a result of improvement in public health standard in recent years in Jordan.

Many factors contribute to the increased incidene of amoebiasis in spring \& summer e.g. increase fly population and food \& water contamination.

The increased incidence of amoebiasis in lower age group (1-10 years) may be explained by the lower 
standard of hygiene in children of that age.

Immunity in older ages may make intestinal mucosa impermissible to proliferation of the organism.

No differentiation between $E$. histolytica \& E. dispar was performed. In fact, many studies have found that most organisms labelled as E.histolytica were E.dispar.

Differentiation is done with modified $\mathrm{PCR}^{10}$ which was beyond our means.

An important protozoon infection (Cryptosporidium parvum) was not included in this study. Attention should be paid to this parasite in future studies.

\section{REFERENCES}

1- World Health Organization (WHO), Intestinal Protozoan and Helminthic Infection, Technical Report Series No. 666, Geneva (1981).

2- P. C. Beaver, R. C. Jung and E. W. Cupp, Clinical Parasitology, $9^{\text {th }}$ Ed., Lea \& Febiger, Philadelphia, 1984, p. 104.
3- J. E. Alicata and S. W. Dajani, Am. J. Trop. Med. Hyg, 4, 1037 (1955).

4- A. B. Al-Lahham, M. Abu-Saud and A. A. Shehabi, J. Diarrhoeal Dis. Res., 8, 160 (1990).

5- T. E. Na'was and M. N AboShehada, ibid., 9, 305 (1991).

6- J. K. Magambo, E. Zeyhle and T. M. Wachira, East Afr. Med. J., 75, 288 (1998).

7- G. F. Araj, N. Y. Abdul Baki, M. M. Hamze, S. Y. Alami, R. E. Nassif and M. S. Naboulsi, J. Med. Liban., 44, 129 (1996).

8- A. M. Amin, J. Egypt. Soc. Prasitol, 27, 817 (1997).

9- F. S. Al-Joudi and A. M. Ghazal, Bull. Pharm. Sci, Assiut University, 28, 277 (2005).

10- H. Hooshyar, M. Rezaian and B. Kazemi, Ann. Saudi. Med., 23, 363 (2003). 